\title{
Ant Colony Optimization and Analysis of Time Step Resolution in Transmission Expansion Computations for Wind Power Integration
}

\author{
Ida Fuchs, Student Member, IEEE, Terje Gjengedal, Senior Member, IEEE
}

\begin{abstract}
This paper investigates the necessary time step resolution in a transmission expansion planning algorithm based on ant colony optimization. A maximal and efficient wind power integration is the main motivation for the grid expansion, which requires a planning tool dealing with wind energy fluctuations. For the analysis a power system model for the Nordic area is used, including a wind power production scenario for 2030. A comparison of simulation results with different time step resolutions, including average and peak values for wind power production, is presented in this paper.
\end{abstract}

Index Terms--Ant colony optimization, computational intelligence, wind power integration, transmission expansion, Monte Carlo, parallel computing

\section{NOMENCLATURE}

$B^{\text {con }} \quad$ The $R$ branches which are congested most of the time of a year in the reference case

$C_{k, h}^{o} \quad$ System cost for feasible hour $h$ for solution $k$

$C_{k, h}^{\mathrm{P}} \quad$ Penalty cost for infeasible hour $h$ for solution $k$

$C_{k}^{\mathrm{S}_{\text {total }}} \quad$ Total system costs for one year for solution $k$

$C^{\mathrm{S}_{\text {total, ref }}} \quad$ Total system costs for one year for the reference case (without any reinforcements)

$C_{k}^{\mathrm{I}_{\text {annuity }}} \quad$ Annuity of total investment costs for solution $k$

$e \quad$ Index for the outer iterations (expedition)

$E \quad$ Maximal number of expeditions

$h$ Index for the number of calculations for the DC optimal power flow [1...H]

$H \quad$ Maximal number of calculations $h$

$i \quad$ Branch number

$k \quad$ Index for an arbitrary solution $[1 \ldots K]$

$K \quad$ Maximal number of possible solutions

$m \quad$ Index for the inner iteration (mission)

$M \quad$ Maximal number of missions

$n \quad$ Number of branches receiving reinforcements

$q_{k} \quad$ Solution quality of solution $k$

$R \quad$ Maximal number of branches chosen for solution $S_{k}$

I. Fuchs is with the Department of Electric Power Engineering, Norwegian University of Science and Technology (e-mail: ida.fuchs@ntnu.no).

T. Gjengedal is with the Department of Electric Power Engineering, Norwegian University of Science and Technology, and the Norwegian TSO Statnett (e-mail: terje.gjenedal@statnett.no).
$S \quad$ All possible combinations of the branches $s_{i}$ which can receive reinforcements

$S_{k} \quad$ Solution $k$ consisting of $R$ branches $s_{i}$

$S_{i} \quad$ Partial solution branch $i$ belonging to $S_{k}$

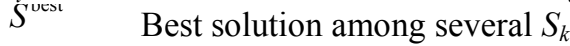

$\varphi^{\text {iuar }} \quad$ Local pheromone reduction rate $(0 \ldots 1]$

$\varphi^{\text {suvar }} \quad$ Evaporation rate (global pheromone reduction rate)

$\tau_{i, m} \quad$ Amount of pheromone for branch $i$ in $m$

$\tau_{i, e} \quad$ Amount of pheromone for branch $i$ after one expedition $e$ is finished

$\tau^{\text {quant }} \quad$ Pheromone quant, the smallest unit of pheromone

$\Delta \tau_{i, e} \quad$ Amount of pheromone for branch $i$ received in $e$

$\Delta \tau_{i}^{\text {start }} \quad$ Amount of pheromone distributed for the branches with the highest Lagrange multipliers in the base case

\section{INTRODUCTION}

$\mathrm{A}_{\mathrm{e}}^{\mathrm{s}}$ significant number of new methods for transmission expansion planning (TEP) have been proposed and tested by researchers and transmission system operators (TSO). It is still a task to meet particular challenges with TEP problems, especially when it comes to coordinate new transmission lines for wind power integration. The computational intelligence method ant colony optimization (ACO) shows a very good capability to deal with highly combinatorial and computational complex problems [1], [2]. The aim of this work is to develop a TEP tool, based on ACO, which is flexible, fast and capable to deal with large system analyses. This paper concentrates on decreasing the necessary calculation time by increasing the time step size, while retaining the same solution quality.

\section{ANT COLONY OPTIMIZATION}

The ACO algorithm has been developed in 1992 [3]. It belongs to the group of swarm intelligence optimizations among computational intelligence methods, which is receiving increased attention with the growing availability of fast computing. ACO algorithms show a positive feedback in solution recovery. They are based on a distributed computation, which avoids premature convergence and use a constructive heuristic to find acceptable solutions in an early stage of the search process [4]. In addition the algorithm is very flexible and makes it possible to use parallel computing 
saving tremendous amounts of calculation time, which is a major advantage when dealing with large simulation.

\section{A. Inspiration from nature}

The idea behind ACO is the behaviour of ants in ant colonies. When a single ant finds food, it travels back to the nest and leaves a serum called pheromone on its path. Thus, other ants find the way to the food source by following the pheromone path. Due to evaporation of the pheromone the following ants will not exactly walk the same path as the first ant. After a certain time interval there will be several paths with different pheromone concentration levels. Subsequently the shortest path will be travelled by most ants and hence receive the highest pheromone concentration. Finally all ants travel the shortest path between food source and nest.

\section{B. Mathematical formulation of the model}

The main characteristic for ACO is a solution quality dependent memory of good candidates being capable to solve the optimization problem. The memory is created by a probability array based on the phenomena of marking paths with pheromone. The amount of pheromone defines the probability value for good solutions in the solution set. The pheromone update can be done in different ways, depending on the version of ACO. Ever since the first ant colony algorithm was published in 1992, the method has been improved continuously providing various versions of ACO. The ant colony system (ACS) shows the best performance for solving different computationally intensive combinatorial problems thus was chosen for this work [5].

The ACS consists of two pheromone update rules. The local pheromone update rule has the main aim to diversify the search performed by subsequent ants during iterations. This is achieved by pheromone reduction on the local, but not on the global level. After one mission $m$ has been successfully fulfilled by one ant forming a solution $S_{k}$, the pheromone reduction rule is applied to any partial solution $s_{i}$ that was chosen by that ant:

$$
\tau_{i, m+1}=\left\{\begin{array}{cc}
\varphi^{\text {local }} \cdot \tau_{i, m} & \text { if } s_{i} \in S_{k} \\
\tau_{i, m} & \text { otherwise }
\end{array}\right.
$$

where $\tau_{i, m}$ is the pheromone amount at $s_{i}$ and $\varphi^{\text {local }}$ is the local pheromone reduction rate.

The global, also called offline, pheromone update rule is applied for the best solutions found during the iteration. This can be done in several ways e.g. update the iteration-best or the best-so-far solution. If $s_{i}$ is part of the best solution $S^{\text {best }}$, it receives pheromone according to the following rule:

$$
\tau_{i, e+1}=\left\{\begin{array}{cc}
\varphi^{\text {global }} \cdot\left(\tau_{i, e}+\Delta \tau_{i, e}\right) & \text { if } s_{i} \in S^{\text {best }} \\
\varphi^{\text {global }} \cdot \tau_{i, e} & \text { otherwise }
\end{array}\right.
$$

where $\varphi^{\text {global }}$ is the global pheromone evaporation rate and $\Delta \tau_{i, e}$ is the amount of pheromone received in iteration $e$.

Controlling the amount of pheromone used for the update is one of the main challenges when developing ACO algorithms. When ants walk paths the amount of pheromone grows faster on shorter paths, only because more ants manage to walk shorter paths during the same time period as on longer paths. This effect is represented by determining the solution quality, e.g. difference in path length, time or cost savings.

At the end of each iteration the pheromone of every $s_{i}$ is multiplied by the evaporation rate, which represents the fact that pheromone disappears over a certain time period. From a mathematical point of view this means that solutions which are not visited anymore will acquire a decreasing probability to be chosen.

\section{Analogy for transmission expansion planning}

For TEP the following analogies can be constituted. The paths being walked by ants represent the branches which receive reinforcements. The path length from nest to food, being the objective to minimize, is represented by the sum of system and investment costs for the TEP. The system costs are a result from a DC optimal power flow with the implemented reinforcements on the chosen transmission lines.

The path length defining the solution quality is represented by the saving of system costs due to expanding transmission capacity.

The values of pheromone for each branch are multipliers of the smallest pheromone unit, here called pheromone quant, which are related to the number of branches in the model.

\section{TRANSMISSION EXPANSION PROBLEM}

In transmission expansion problems the solution matrix $S$ consists of all possible combinations of branches $s_{i}$ which can receive reinforcements. For one solution candidate $S_{k}$ each branch $s_{i}$ can only be chosen one time to receive reinforcement and the maximal number of allowed reinforcements is $R$.

$$
S=\left[\begin{array}{lllll}
S_{1} & \cdots & S_{k} & \cdots & S_{K}
\end{array}\right]
$$

$K$ is the number of possible combinations, defining all potential solution candidates. It is determined by the number of existing branches $n$ in the model and the number of allowed reinforcements $R$ in the following way

$$
K=\left(\begin{array}{c}
n+R-1 \\
R
\end{array}\right)
$$

The number $K$ can become tremendously large. Considering even a medium sized problem with 44 branches and allowing 10 reinforcements $K$ reaches over 1.9 billion possible combinations. This large number shows that a classical approach of calculating all possible combinations and comparing them is not accomplishable in a reasonable time.

After $M$ solutions are chosen in each iteration, an evaluation of the solution feasibility and quality is performed by a DC optimal power flow based on linear programming. The general mathematical formulation can be found in [6].

The DC optimal power flow is calculated for the relevant 
years and delivers the system cost as optimization result, which is the main input into the objective function for the ACO. The system cost for one hour is defined as the sum of the production of all generators multiplied with their marginal costs. The total system $\operatorname{cost} C_{k}^{S_{\text {toal }}}$ is the sum of system costs for every feasible hour $C_{k, h}^{\mathrm{S}}$ plus a penalty $\operatorname{cost} C_{k, h}^{\mathrm{P}}$ for the hours where the DC optimal power flow could not find a solution, i.e. that the suggested reinforcement plan cannot meet the required capacity during that hour.

$$
C_{k}^{\mathrm{S}_{\text {total }}}=\sum\left\{\begin{array}{lc}
\sum_{h} C_{k, h}^{\mathrm{S}} & \text { if } h \text { is feasible } \\
\sum_{h} C_{k, h}^{\mathrm{P}} & \text { else }
\end{array}\right.
$$

Further results from the DC optimal power flow are the Lagrange multipliers associated with each constraint, especially those according to branch constraints. A high value means reinforcing in the corresponding line would give an equivalent good improvement of the system power flow and so a lower system cost. These multipliers calculated from the base case (without any reinforcements) are used as heuristic information for the ACO algorithm. They define the vector of start pheromone values leading the search in the most significant direction.

The optimization function is defined by the minimization of the total system costs $C_{k}^{S_{\text {total }}}$ for the whole year and the annuity of total investment costs $C_{k}^{\mathrm{I} \text { amuity }}$ for the chosen reinforcement:

$$
\operatorname{Min} f(k)=C_{k}^{\mathrm{S}_{\text {total }}}+C_{k}^{\mathrm{I}_{\text {annuity }}}
$$

The objective of minimizing system costs can be seen as a regulatory authority viewpoint. Minimizing investment costs can represent the system operator viewpoint. Thus, a combination of both would reflect a system with a regulated TSO monopoly.

\section{Methodology}

The complete optimization algorithm for finding the best transmission expansion plan consists of two separate optimization procedures: The ACS, which is searching for solution candidates, and the DC optimal power flow that verifies the feasibility and quality of each candidate.

During the optimization process the ACO algorithm uses different heuristic information to determine the search for solutions. The heuristic information is given by the DC optimal power flow results. The optimal power flow of the base case (system without any reinforcements) delivers the reference value for the system cost $C^{\mathrm{S}_{\text {oualrer }}}$ and the Lagrange multipliers for the branch constraints. These multipliers are used to define the pheromone start value in the following way:

$$
\tau_{i}=\left\{\begin{array}{cc}
\tau^{\text {quant }}+\Delta \tau_{i}^{\text {start }} & \text { if } i \in B^{\text {con }} \\
\tau^{\text {quant }} & \text { else }
\end{array}\right.
$$

$B^{c o n}$ are the branches which are congested most of the time of a year. $\Delta \tau_{i}^{\text {start }}$ refers to a value representing the number of hours of a year, where the corresponding branch $i$ is congested.

After each expedition consisting of $M$ missions, when the ACO has chosen a set of solutions, a DC optimal power flow is performed and the system cost for the corresponding solution is calculated. The difference of the reference system $\operatorname{cost} C^{\mathrm{S}_{\text {totall,cer }}}$ and the calculated system cost $C_{k}^{\mathrm{S}_{\text {total }}}$ for a solution $k$ defines the solution quality $q_{k}$ and thus the amount of pheromone update.

$$
q_{k}=\left\{\begin{array}{cc}
\left(C^{\mathrm{S}_{\text {total, ref }}}-C_{k}^{\mathrm{S}_{\text {total }}}\right) & \text { if } C^{\mathrm{S}_{\text {total,ref }}>C_{k}^{\mathrm{S}_{\text {total }}}} \\
1 & \text { else }
\end{array}\right.
$$

and the amount of pheromone update is defined as:

$$
\Delta \tau_{i, e}=\tau^{\text {quant }} \cdot q_{k} \quad \forall i \in S^{\text {best }}
$$

There are two different types of update rules which can be used, as mentioned earlier. The iteration-best rule updates the best solution of the solution set after each iteration while the best-so-far rule updates the best solution found so far during all iterations done up to that point. The iteration-best update rule avoids early convergence and delivers better results [5]. A probability vector is calculated from the amount of pheromone received, which defines the likelihood for the branch to be chosen during the next expedition. The ACO algorithm is established according to the following steps (Fig.1):

(i) Until the maximum number $M$ of solutions, being chosen during expedition $e$, is reached, do: Pick a solution $S_{k}$ consisting of $R$ branches. Apply the local pheromone update rule according to Eq. (1).

(ii) Calculate the DC optimal power flow $H$ times (a predefined number according to the case which is studied) of each $S_{k}$ found in (i) and find the best solution $S^{\text {best }}$ of that expedition $e$.

(iii) While $e<E$ do the global update rule for all $s_{i}$ according to Eq. (2) and start (i) again, else finish the algorithm and present results. 


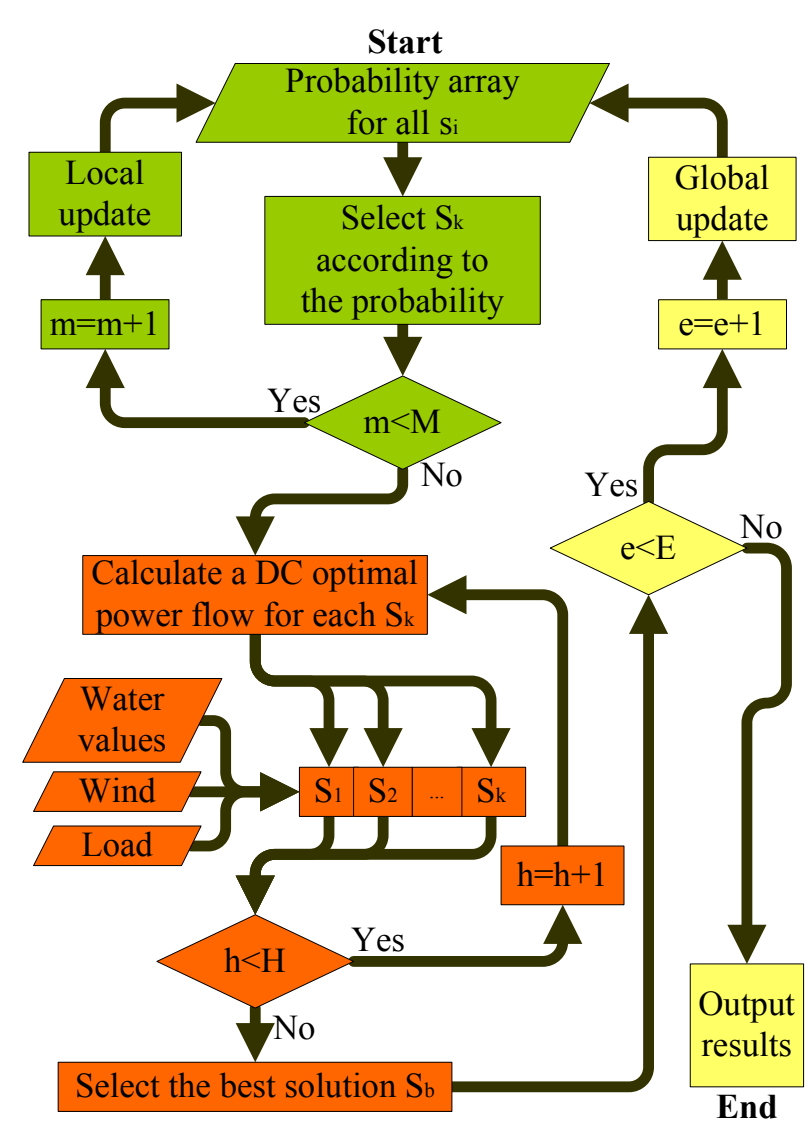

Fig. 1. Flow chart of the total algorithm with step (i) in green, step (ii) in orange and step (iii) in yellow.

The flexibility of the algorithm allows the use of parallel computing, which leads to an enormous reduction of calculation time. The DC optimal power flow, meaning the whole step (ii) in Fig. 1, can be calculated simultaneously for every $S_{k}$ found in step (i), since the input in each single optimal power flow is independent of the output of the other power flows.

The parameter $H$ refers initially to the number of hours for one year. However, in this paper, the number of necessary calculation loops is analyzed by reducing the time step resolution. $H$ will have different values, from 8760 to only 4 , depending on the studied case, which are described in the following section VI.C.

\section{TEST SYSTEM}

The Nordic area was chosen for developing and testing the ACO algorithm. The primary reason is the motivation to develop the algorithm for specific requirements related to that particular system in order to be able to execute more extensive case studies later. Another important reason is the availability of data and information as well as the access to other transmission expansions studies for the Nordic system. It gives the possibility to compare results and define the quality of the performance of the ACO algorithm.

\section{A. Case description}

The Nordic area model has 36 buses, 38 generators, 44 branches, 15 transformers and two voltage levels. The generation is dominated by hydro power. An overview of generation types, number, size and marginal cost intervals can be found in Table I.

TABLE I

GENERATORS IN THE NORDIC MODEL

\begin{tabular}{||l|c|c|c||}
\hline $\begin{array}{c}\text { Generator } \\
\text { type }\end{array}$ & Number & $\begin{array}{c}\text { Aggregated } \\
\text { capacity } \\
(\mathbf{G W})\end{array}$ & $\begin{array}{c}\text { Marginal } \\
\text { cost range } \\
(\boldsymbol{€} / \mathbf{M W h})\end{array}$ \\
\hline Hydro & 15 & 45.8 & $35 \ldots 66$ \\
\hline Fossil & 3 & 22.3 & $25 \ldots 60$ \\
\hline Nuclear & 4 & 11.9 & $10 \ldots 15$ \\
\hline Wind & 16 & 21.9 & 0.5 \\
\hline Total & $\mathbf{3 8}$ & $\mathbf{1 0 1 . 9}$ & $\mathbf{0 . 5} \ldots \mathbf{6 6}$ \\
\hline
\end{tabular}

The optimal power flow uses the generators with lowest marginal costs first. One of the goals of the simulation is a maximum wind power penetration. Thus, wind generators are modeled with very low marginal costs. The hydro generators are simulated with marginal costs according to the weekly average of their water values from 1949 to 1999 related to the northern Europe system [7], [8]. This represents an average year for the hydro power plants and their reservoir level. All generation costs are linear cost functions.

The reinforcement of a branch connotes its maximum capacity is doubled; however the minimal capacity expansion is $500 \mathrm{MW}$. The investment costs depend on the voltage level, the added capacity and a factor defining the infrastructure quality of the branch location receiving reinforcements. Thus, the cost range for the reinforcements lies between 225 and $1000 \mathrm{k} € / \mathrm{km}$. The annuity for the total investment is calculated with standard parameters, i.e. the amortization time is set to 20 years and the interest rate is set to 5 percent.

\section{B. Scenario description}

The scenario refers to the year 2030. The annual load increase was set to 0.5 percent calculated from load data of 2007 according to prognosis done by Statnett [9].

The installed wind power capacity was taken from the medium wind scenario for 2030 [10]. Based on the COSMO EU data set the production is calculated individually for each wind power production facility in the modelling area [11].

The wind generators are forced to run at 100 percent of the wind power production, the nuclear power plants are set to a minimum production of 20 percent of their capacities, while all other generators can be adjusted arbitrarily by the optimal power flow.

\section{Study description}

A smaller time step allows more detailed information about wind power production, but causing high calculation times. To analyze that fact, it is possible to reduce the number of calculation loops by reducing the number of calculated hours. In this study, eight different cases were chosen: 
a) Hourly time step (HTS)

b) Weekly time step (WTS)

c) Monthly time step (MTS)

d) Peak monthly time step (PMTS)

e) Seasonal time step (STS)

f) Monte Carlo - 4000 hours (MS4000)

g) Monte Carlo - 1000 hours (MS1000)

h) Monte Carlo - 100 hours (MS100)

In HTS an hourly time step is used, which is the initial case, here used as reference case. In WTS weekly average values and in MTS monthly average values are used. In PMTS and STS the hour with the highest wind power production is used respectively for each month or each season. The Monte Carlo simulations pick random hours from the whole year. The comparison includes 4000, 1000 and 100 arbitrary hours. The aim is to analyze which method (average, peak or random hours) and which resolution of data can produce the best results while saving calculation time. Table II shows more details about the different cases.

TABLE II

STUDIED CASES

\begin{tabular}{||c|c|c||}
\hline $\begin{array}{c}\text { Case } \\
\text { name }\end{array}$ & $\begin{array}{c}\text { Resolution of } \\
\text { data }\end{array}$ & $\begin{array}{c}\text { Number of calculations } \\
\text { during one simulation year }\end{array}$ \\
\hline HTS & Hourly & 8760 \\
\hline WTS & Weekly & 52 \\
\hline MTS & Monthly & 12 \\
\hline PMTS & Hourly & 12 \\
\hline STS & Hourly & 4 \\
\hline MS4000 & Hourly & 4000 \\
\hline MS1000 & Hourly & 1000 \\
\hline MS100 & Hourly & 100 \\
\hline
\end{tabular}

\section{Parameter definition}

The parameters for the ACO algorithm are set to the following values as shown in Table III. The smallest amount of pheromone update, the pheromone quant, must be dependent on the system size, in particular on the number of branches. A reasonable number is 10 percent of the number of branches. The number of reinforcements is set to maximal 10 , meaning that the algorithm can choose to expand any number between 0 and 10 .

TABLE III

ACO PARAMETERS

\begin{tabular}{||l|c||}
\hline \hline Parameter & Value \\
\hline Pheromone quant $\tau_{q}$ & 4.5 \\
\hline Reduction rate $\varphi_{l}$ & 0.8 \\
\hline Evaporation rate $\varphi_{g}$ & 0.8 \\
\hline Number of reinforcements $R$ & 10 \\
\hline Number of missions $M$ & 8 \\
\hline Number of expeditions $E$ & 100 \\
\hline \hline
\end{tabular}

\section{RESULTS}

The algorithm was implemented in Matlab R2010a, using Matpower 4.0 [6]. All calculations are done with a 4 x AMD Opteron 6174 with $2.2 \mathrm{GHz}$ processors, each with 12 cores, adding up to a total of 48 cores. Matlab's Parallel Computing Toolbox 5.0 was used to perform the inner loop (Fig. 1, (ii)) of the optimization process.

\section{A. Presentation}

Table IV shows the simulation results for the different cases described previously. The objective function value is the sum of system cost, penalty cost for infeasible hours and investment cost, altogether according to Eq. 5 and Eq. 6. The system cost reduction in percent illustrates the improvement of the system optimality by investing in the proposed new transmission lines. The number of infeasible hours refers to the remaining hours where the optimal power flow, including the best transmission expansion plan, could not find a solution. This is primarily caused by high wind power production.

TABLE IV

CASE RESUlTS

\begin{tabular}{||l|c|c|c|c||}
\hline $\begin{array}{l}\text { Case } \\
\text { name }\end{array}$ & $\begin{array}{c}\text { Objective } \\
\text { function } \\
\text { value } \\
\mathbf{( k \ell / h )}\end{array}$ & $\begin{array}{c}\text { System } \\
\mathbf{c o s t} \\
\text { reduction } \\
\mathbf{( \% )}\end{array}$ & $\begin{array}{c}\text { Infeasible } \\
\text { hours } \\
\text { per year }\end{array}$ & $\begin{array}{c}\text { Calculation } \\
\text { time } \\
\mathbf{( h )}\end{array}$ \\
\hline HTS & 713 & 17 & 71 & 19.6 \\
\hline WTS & 737 & 15 & 407 & 0.6 \\
\hline MTS & 804 & 7 & 1605 & 0.5 \\
\hline PMTS & 721 & 17 & 149 & 0.5 \\
\hline STS & 715 & 17 & 67 & 0.5 \\
\hline MS4000 & 727 & 16 & 239 & 10.4 \\
\hline MS1000 & 726 & 16 & 198 & 2.8 \\
\hline MS100 & 740 & 14 & 448 & 0.8 \\
\hline \hline
\end{tabular}

Figure 3 shows which transmission lines were reinforced in the two cases with the best objective function values, which are HTS and STS. The optimal solution is very similar in the two cases.

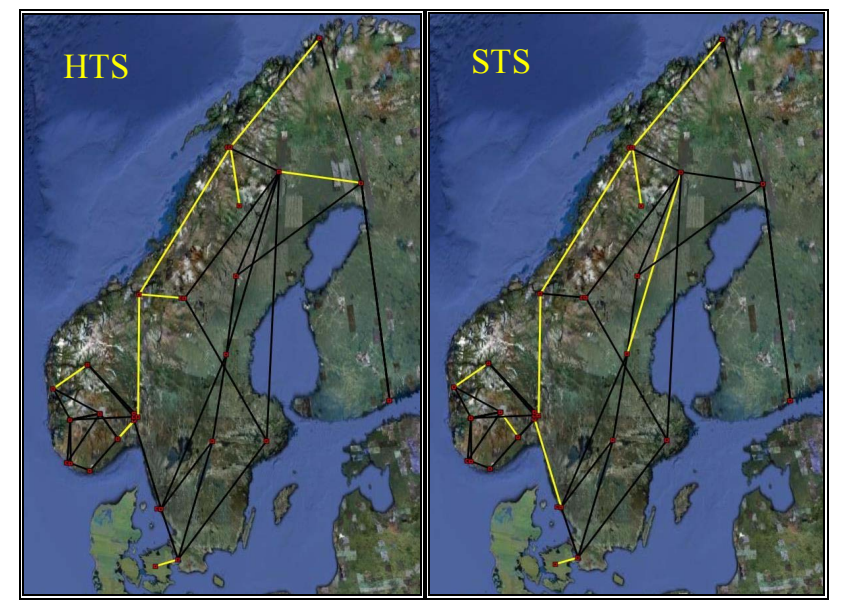

Fig. 2: Best transmission expansion plans for simulation cases HTS and STS. The optimal solution is highlighted yellow. 


\section{B. Discussion}

The best solution according to the objective function value was found by HTS. Nevertheless, the results for both PMTS and STS are almost as good as the calculation for every hour, but with a tremendous time saving advantage (Fig. 3). Instead of almost 20 hours of calculation time, the PMTS and STS can deliver the same results in just half an hour.

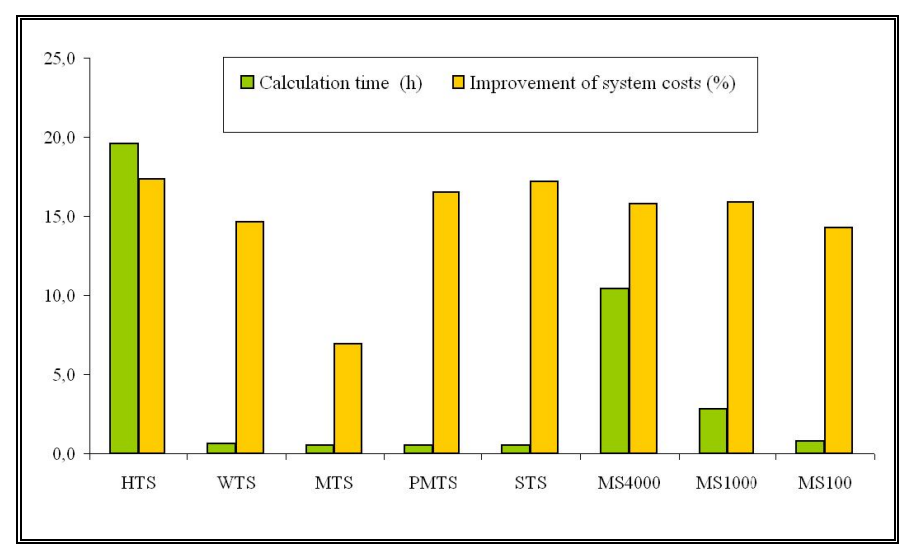

Fig. 3: Calculation time (green) in hours and improvement of system cost (yellow) in percent for the studied cases with different time step resolution.

For the both PMTS and STS the wind power production peak hours were used and therefore the main criteria for the ACO. Hours with maximal wind power production are leading the heuristic search included in ACO in the same direction as the simulation of the full year with 8760 calculation hours.

The cases with average data, including WTS and MTS had a tendency to reduce the number of reinforcements. In both cases the ACO chose to expand 5 instead of 10 branches. From the objective function point of view, this reduces the investment cost and should increase the penalty cost for infeasible hours. These are first calculated at the end of the simulation process, since the search is based on average data. That fact leads the search process based on average data into pseudo-optimal directions.

\section{CONCLUSION}

The analysis in this paper shows that with the given parameters and settings of the ACO algorithm, the optimal time resolutions for the simulation are monthly or seasonal wind power production peak hours. These lead the search in the optimal direction and deliver good results with high quality in short calculation times. Simulations based on average data or arbitrary hours chosen by a Monte Carlo simulation tend to lead the search process in pseudo-optimal direction.

\section{REFERENCES}

[1] A. Silva et al, "Reliability worth applied to transmission expansion planning based on ant colony system", Electrical Power and Energy Systems, Vol. 32, pp. 1077-1084, 2010

[2] I. Fuchs et al, "Transmission expansion planning in the Nordic system for wind power integration based on ant colony optimization", OFFSHORE, Amsterdam, 2011
[3] M. Dorigo, "Optimization, learning and natural algorithms", $\mathrm{PhD}$ thesis, Politecnico di Milano, 1992

[4] D. Saxena et al, "Application of computational intelligence in emerging power systems", International Journal of Engineering, Science and Technology, Vol. 2, No.3, pp 1-7, 2010

[5] M. Dorigo et al, "Ant Colony Optimization - Artificial Ants as a Computational Intelligence Technique", IEEE Computational Intelligence Magazine, pp. 28-39, Nov. 2006

[6] R. D. Zimmerman et al, "MATPOWER Steady-State Operations, Planning and Analysis Tools for Power Systems Research and Education," Power Systems, IEEE Transactions on, vol. 26, no. 1, pp. 12-19, Feb. 2011

[7] O. Wolfgang, A. Haugstad, B. Mo, A. Gjelsvik, I. Wangensteen, G. Doorman, "Hydro reservoir han-dling before and after deregulation", Energy, Vol. 34, pp. 1642-1651, 2009

[8] S. Jaehnert et al, "Modelling an integrated northern European regulating power market based on a common day-ahead market", Proc. of IAEE International Conference, Rio de Janeiro, Juni 2010

[9] Statnett, "Netutviklingsplan", www.statnett.no

[10] G. Van der Toorn, et al and Partners Ltd, "EU TradeWind Project, Work Package 2: Wind Power Scenarios, WP2.1: Wind Power Capacity Data Collection", 2007, www.trade-wind.eu

[11] T. Aigner et al, "Modelling wind power production based on numerical prediction models and wind speed measurements", PSCC2011, Stockholm, 2011

\section{BIOGRAPHIES}

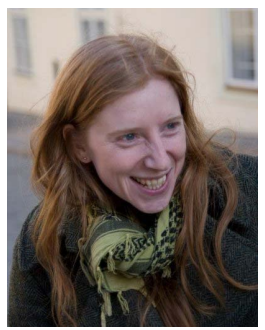

Ida Fuchs was born in Grossenhain in the Freistaat Sachsen in Germany, on February 3th, 1981. She graduated from the Werner von Siemens Gymnasium, Grossenhain, and studied at the Technical University of Chemnitz, Germany. She received her Diploma in Industrial Economics with Electrical Engineering in 2006 and a Pre-Diploma in Physics in 2005.

During her master thesis in 2006 she was involved in the world's first autonomous energy supply system combining wind power and hydrogen (including electrolysis and fuel cell technology) on the Norwegian Island Utsira.

Her employment experiences include Norsk Hydro Production AS Research Center (2006), Conergy AG (2004), Norsk Hydro ASA (2003) and EnviaM RWE Group (2001). Currently she is employed at Norwegian University of Science and Technology in Trondheim, Norway, working with transmission expansion planning for wind power integration.

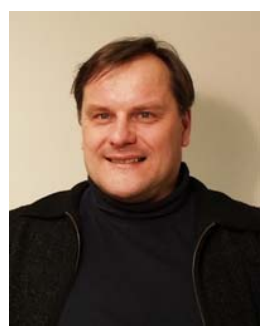

Terje Gjengedal was born in Sandane in 1958 . $\mathrm{He}$ received a MSc and a Phd in electrical engineering from the Norwegian University of Science and Technology in 1983 and 1987 respectively. He has been working as a senior research scientist, and a visiting professor in the US. While vice president of Statkraft he was working on technology, system and market integration of wind power and other renewable sources. Since 2009 he is the R\&D Director at Statnett. Besides he holds a professor position at the Norwegian University of 